\title{
An Algorithm of Fuzzy Inference System for ISAR Image Classification
}

\author{
Krasimir Slavyanov, Chavdar Minchev \\ National Military University, Faculty of Artillery, Air Defense and Communication and Information Systems. \\ Address: Karel Shkorpil 1, Shumen, BG-9700, Bulgaria.
}

\begin{abstract}
This article offers an original ISAR image classification procedure based on Mamdani fuzzy inference system (FIS) dedicated to compute multiple results each from different type of analyzing criteria. The modeling and information analysis of the FIS are developed to draw a general conclusion from several results each produced by classification from neural network. Simulation experiments are carried out in MATLAB environment.
\end{abstract}

Keywords: fuzzy inference system, membership function, fuzzy rule.

\section{INTRODUCTION}

For all Inverse Synthetic Aperture Radar (ISAR) systems high performance and better image reconstruction technologies are vital. An opportunity for improved information analysis in that area is suggested to be the development of better algorithms for recognition of the various flying objects. To solve the classification problem after the optimization procedures of a radar image a fuzzy logic algorithm can be used to maximize the recognition rate while controling the error rate [1], [2]. Fuzzy logic can be applied also to the identification "friend-or-foe" airplanes using hybrid algorithm combining fuzzy neutral network with a probability factor. A hybrid algorithm combining fuzzy neutral network with probability factor (FNNP), multi-level fuzzy comprehensive evaluation and the Dempster-Shafer (D-S) theory can be implemented too [3].

In other fields of science the determination of the specific rules in classification system with fuzzy logic can solve problems in the classification of vessels caused by the insufficient amount of information on one SAR channel [4]. Nowadays the improved modeling and information analysis of ISAR systems are developed by implementation of detailed 3D model of observed flying object [5]. The neural network procedures can be applied to classify different features of the objects in the ISAR image as well as to compare the ISAR images with some known aircraft models in a specific database [6].

\section{PRECONDITIONS AND MEANS FOR RESOLVING THE PROBLEM}

Preconditions.

A specific experimental arrangement is developed for the proposed algorithm of fuzzy inference system for ISAR image classification. The arrangement consists of five neural networks each working with a specific database of models after the optimization procedures of the ISAR image. One neural network is designed to classify the object in the ISAR image by its size (small, middle or big). Another - computing the jets position (rear, wing or integrated in the fuselage), third - comparing the image with database of 16 aircraft models. Fourth comparison is made for contour of the ISAR image with a database of 16 aircraft contour models. The fifth neural network is for contour ratio comparison evaluating the number of the corresponding pixels with the contour images database. Each neural network can be developed in MATLAB environment in order to produce a result value between zero and one.

The linguistic variable is a main term in the fuzzy logic and is described a variable, witch value defines a set of verbal characteristics of a feature [7].

Building a system for making a final decision based on fuzzy logic.

The tools of fuzzy logic allows the use of two approaches to implement a system for decision. Various membership functions - functional relationships that determine the way in which each point of entrance area (input variables) form the baseline background (degree of affiliation) within the range of zero to one for the membership functions of the output variables.

In this fuzzy inference system the use of membership functions of Gaussian type are chosen, subject to the following factors: the specificity of recognition of images; universality of application of Gaussian functions; availability of similarity in model airplanes; evenness of the form; pronounced maximum; values other than zero for all points. Gaussian curves are subdivided into two types according to their form: a simple Gaussian curve and the two-way combination of two different Gaussian http://dx.doi.org/ 10.17770/etr2017vol2.2599 
curves. Similar to them is the function of belonging of type "bell" defined by three parameters (Fig. 1).

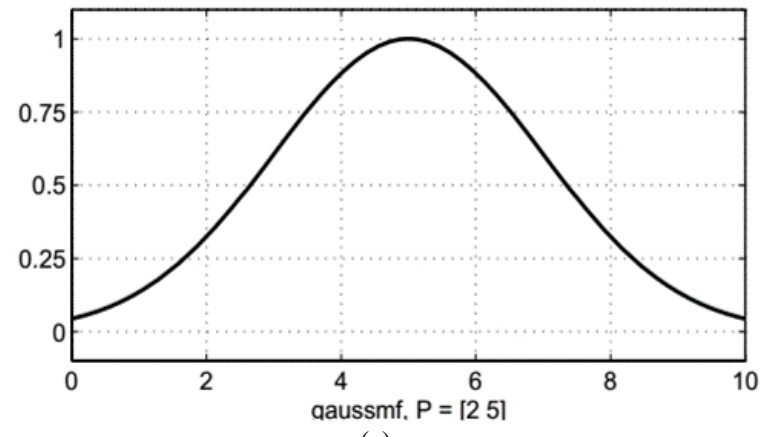

(a)

(b)
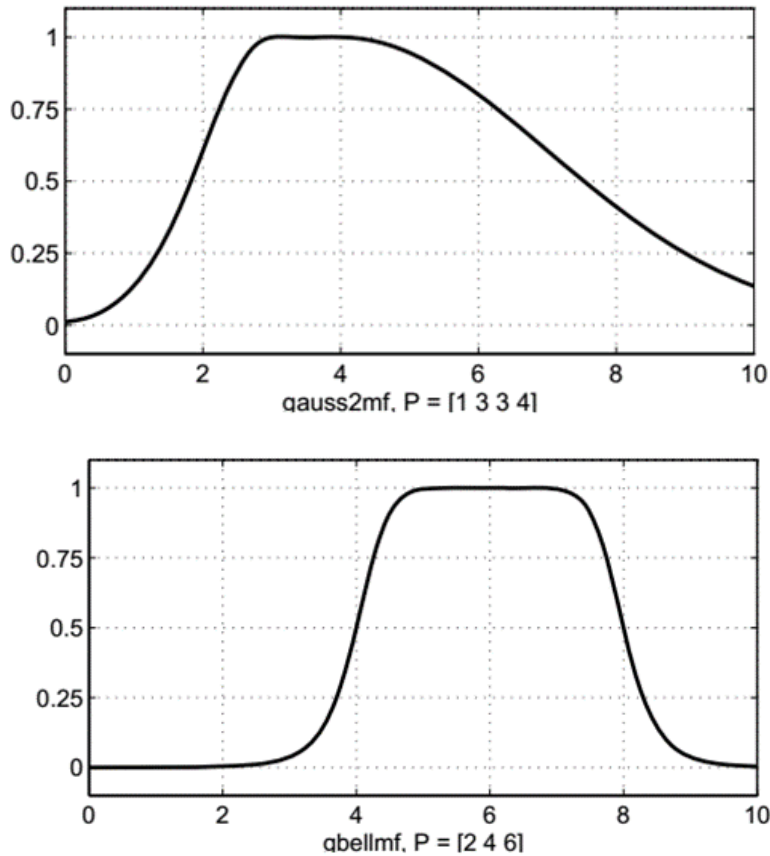

(c)

Fig.1 Graphical description of the functions of belonging to the Gaussian distribution - plain (a) combination (b) and type "bell" (c).

The degree of membership of an object to the structure of fuzzy membership functions is determined by the value of membership ranging from zero to one. Thus the membership function associated with a fuzzy set of inputs, is used to position the output value in the corresponding area of the membership.

Mamdani's method is commonly used method for decision-making with fuzzy logic [8]. The method is based on the classic staging of Lotfi A. Zadeh [9, 10]. In Mamdani the output membership functions are expected to be fuzzy sets. After a merging of the different results is necessary fuzzy set for each output variable to be converted to a number.

To form a final decision in recognition system proposed in this thesis was chosen method of Mamdani. This method is preferable to the method of Sugeno because the demand system for decision making with fuzzy logic and the specifics of outcomes in identifying, are corresponding with the characteristics of this method.
The aggregation function for the results is selected to be one that would seek the maximum value in each membership function to the input fuzzy variables.

Given that demand maximum similarity to a reference model is characteristic for each of the built and trained neural networks function is selected to form the final result the type "largest of maximum" as a defuzzification. The Described parameters of demand system for decision making with fuzzy logic are applied in the implementation of fuzzy logic summarizing the results of the neural networks.

The system is built with the tools of Matlab and is depicted in Fig. 2.

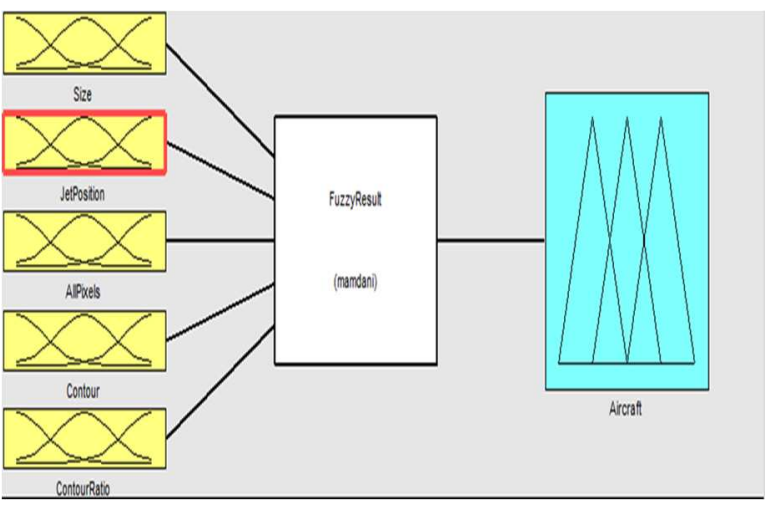

Fig. 2. Block diagram of the system for decision making by means of the Mamdani method, built in Matlab.

The input variables on the left side of the system are five corresponding to each of the five neural networks used to classify the object in the resulting image of ISAR. Each of these input variables is made of such membership functions as are the classes of the outputs of the neural network. The output variable is a synthesis of all the rules applied to the input variables and is the object of recognition that best satisfied these fuzzy rules.

The chosen shape of the curve membership function to each fuzzy set is Gaussian combination membership function. The results of the first neural network should fall within three fuzzy sets defined by the functions of belonging in the range of 0 to 3 , as sectors $0-1,1-2$ and 2-3 are distributed in the same sequence as the output values of the first neural network (small, middle and big). The specific form of these functions is tailor made to take maximum space in the range 0-3 to fall into the fuzzy sets higher percentage results from the input value. The shape responds to the need with the increase of the coefficient of similarity between the subject and a reference model (object size) to increase the degree of belonging to the elements of the fuzzy set (Fig. 3).

Similar are the parameters of input variable for the results of the second neural network, analyzing the position of engine for the object of recognition, because here the neural network result is designed to have three values again. For input variable with the 
result of the recognition of the object in his thick silhouette 16 fuzzy sets are created corresponding to the number of reference solid models in the third neural network.

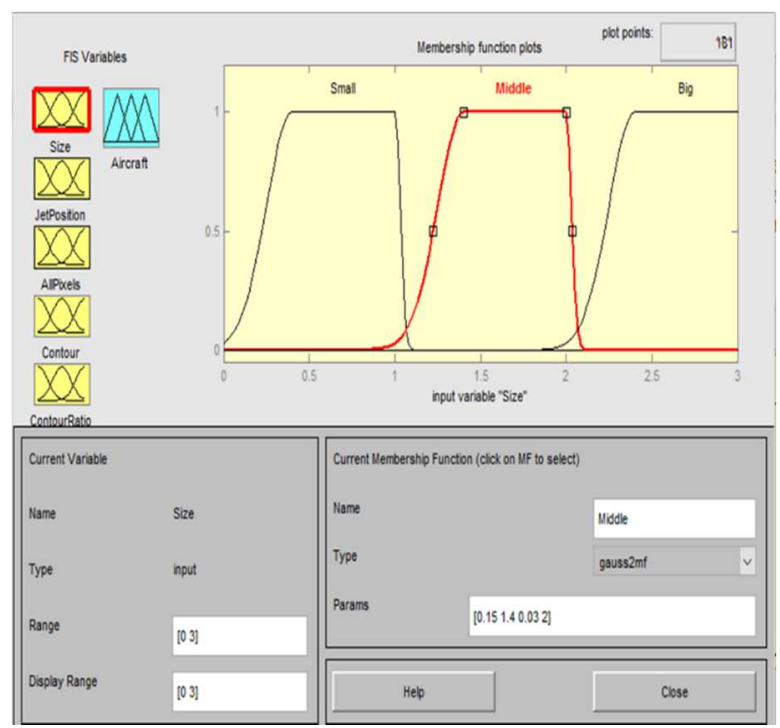

Fig. 3. Graphical expression of membership functions of fuzzy sets corresponding to the size of the object.

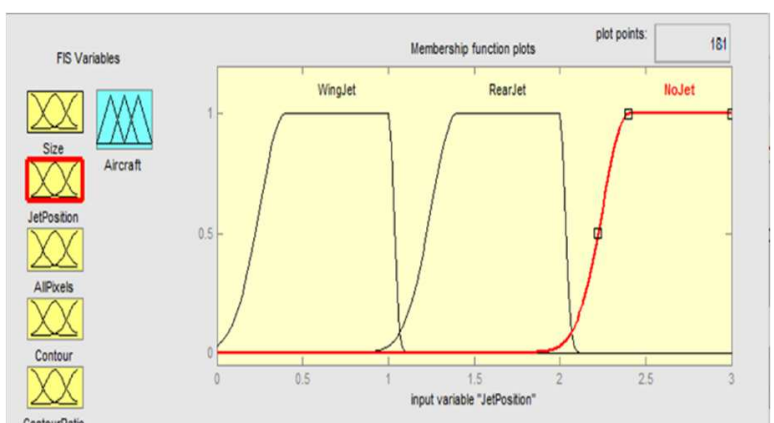

Fig. 4. Diagram describing the membership functions of fuzzy sets corresponding to the variation in position of the engine, result of neural network

The same logic is applied with Gaussian combination membership function and spaces with a maximum surface area, respectively, membership functions results in ranges $0-1$ on the outcome of the first reference model, 1-2 - the second, etc. filling the interval 0-16 (Fig. 5).

Analogous is the structure of input variables corresponding to the result for contour of the object and the result for the contour ratio comparison produced from the dedicated neural networks four and five. In this scientific work they are not described in detail.

The output fuzzy variable is made up of fuzzy sets, broken again in the range $0-16$, given the objects in the database that seek similarities to object recognition. The membership functions with the Gaussian distribution of type "bell" are aimed to summarize at maximum the results of the input variables, classified by the rules. Membership functions for the fuzzy sets of the output variable are shown in Fig. 6. The particular form aims to distinguish in maximum the membership functions of each model for comparison. The shape is consistent with the selected function to summarize the results that is formed by the maximum value in each membership function of the input fuzzy variables.

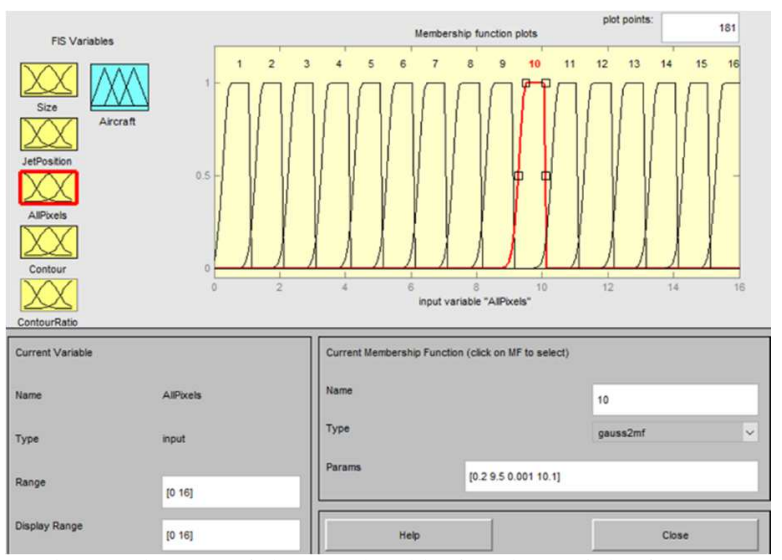

Fig. 5. Diagram of the membership functions of the fuzzy sets corresponding to each solid etalon model and the number of result values of the third neural network.

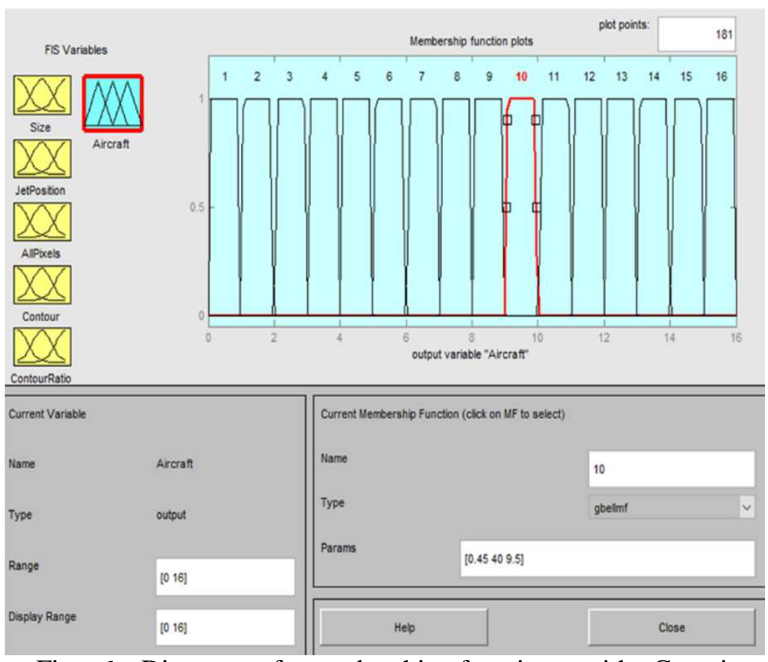

Fig. 6. Diagram of membership functions with Gaussian distribution type "bell" fuzzy sets of the output variable corresponding to the number of reference models used for object recognition in various indicators.

The set of rules necessary for the operation of the system is composed of 32 rules divided into 2 groups, the weight of every rule of the groups is equal to one.

For the first set of rules for each reference model are selected functions of fuzzy sets of input variables that describe it in its relevant characteristics (size, engine position, solid shape, contour, the number of matched pixels to the total number of pixels for the outline model) as described classes at the output of each of the neural networks. The operation used for the various fuzzy sets which are obtained for each criterion for comparison is a logical "AND" to reflect the intersection of these fuzzy sets and to comply thus with the result of each neural network. Rules are 16 in number and one of them is used for example: 
(1.) (Size $==$ "big ") $\cap($ Jet possition=="wing ") $\cap$ (Solid shape $==$ "C-130 H“) $\cap($ Contour $==" C$ $\left.130 \mathrm{H}^{\text {") }}\right) \cap\left(\right.$ ContourRatio $==$ "C $\left.-130 \mathrm{H}^{\text {" }}\right)=>$ (Object $==$ " $\left.\mathrm{C}-130 \mathrm{H}^{\text {") }}\right)$

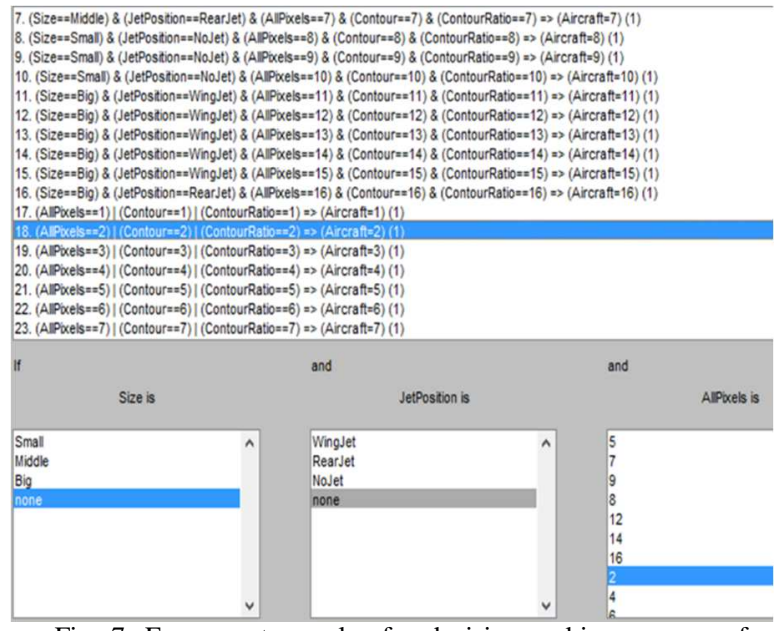

Fig. 7. Fuzzy system rules for decision making process of a system with fuzzy logic simulated in Matlab.

The second group of 16 rules are designed to treat the results of neural networks that specify the similarity of objects with specific reference model, i.e. these are three neural networks (Solid shape, Contour and ContourRatio). In the rules the logical operator "OR" is used for operation between the selected fuzzy sets, thereby to address potential similarities of objects with different reference models in various indicators of comparison (Fig.7). According the description, the set of 16 rules adopted in that group can be explained, with the example:

\section{(2.) (Solid shape $==$ "Rafale") $\cup$ (Contour $==$ " Rafale“) $\cup$ (ContourRatio $==$ "Rafale“ $)=>$ $=>\left(\right.$ Object $==$ " Rafale $\left.^{\text {" }}\right)$}

The described fuzzy inference system is aimed to summarize the results of five neural networks that have output variables of different nature, to put the fastest result as a digital value corresponding to the combination of degrees of matching the object of recognition with the different reference models.

\section{RESULTS AND DISCUSSION}

Numerical experimental results of observed object classification by neural networks and deciding upon its recognition using fuzzy logic.

The results of numerical simulation experiment of observation with reference model aircraft Rafale is described on Fig. 8 as follows:

- In section (a) is illustrated the neural networks operation;

- In section (b) is is depicted the result of decision making process with fuzzy inference system (FIS), the
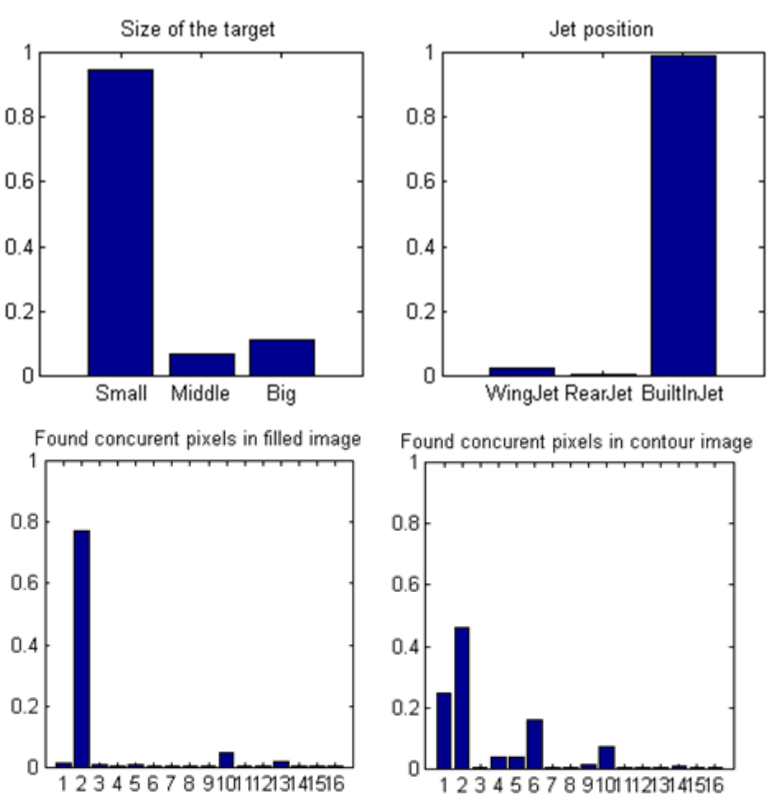

(a)

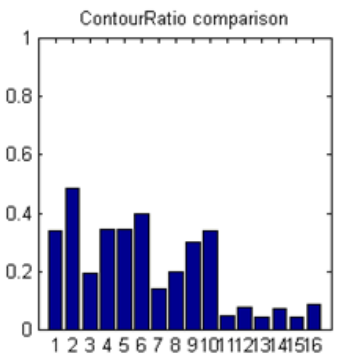

(b)

Fig.8. Graphically expressed results of the procedure for recognition. Score 1.76 (b) is connected to the output variable with the number 2. The object in that case is recognized as the plane Rafale.

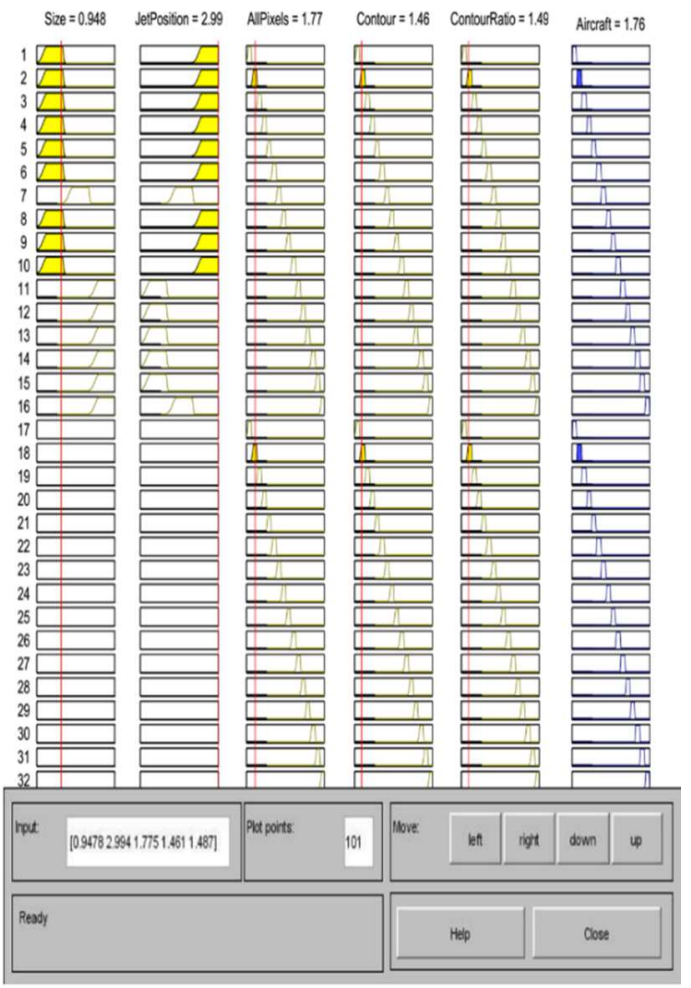


Environment. Technology. Resources, Rezekne, Latvia Proceedings of the $11^{\text {th }}$ International Scientific and Practical Conference. Volume II, 154-158

entrance to it is presented with the five neural networks results as a fuzzy sets and the value of the parameter Aircraft is used to describe the number corresponding to the FIS decision - 1.76 The result is related to the output variable with this number. The object is recognized as the aircraft Rafale.

\section{CONCLUSION}

A system for deciding fuzzy aimed to summarize the results of all neural networks in accordance with a system of logical rules is established. The membership sunctions of all input variables, are used to formulate a reasoned conclusion, despite the different nature of their dimensions. One of the problems inherent in the operation of neural networks is permitted, they are able to produce a satisfactory result, but not to formulate a conclusion. Flexible approach in formulating decisions is of particular importance in mind the possible application of the developed recognition system.

By the implementation of the combination of rules for the operation of the system with fuzzy logic the computational burden in making the final decision is reduced and the rapid adaptation to changes in the composition and nature of neural networks is allowed. The system can be used for image recognition obtained in the radar operating on the principle of inverse aperture synthesis.

\section{REFERENCES}

[1] Boulay T., Lagoutte J., Mohammad-Djafari A., Gac N., A FuzzyLogic Based Non Cooperative Target Recognition, Signal Image Technology and Internet Based Systems (SITIS), 2012 Eighth International Conference, Nov. 2012.. pp. 410-415.

[2] M. V. Vasileva, „Fuzzy sets. Theory and practice“, ISBN 978954-9681-33-8, 2008.

[3] Tan Y., Yang J., Li L., Xiong J., Data fusion of radar and IFF for aircraft identification, Journal of Systems Engineering and Electronics ( Volume: 23, Issue: 5, Oct. 2012 ), 2012, pp. 715 722.

[4] Margarit G., Tabasco A., Ship Classification in Single-Pol SAR Images Based on Fuzzy Logic, IEEE Transactions on Geoscience and Remote Sensing (Volume: 49, Issue: 8, Aug. 2011), pp. $3129-3138$.

[5] Minchev, C.N., Slavyanov, K.O., An opportunity for improved modeling and information analysis in ISAR systems, "Machines, Technologies, Materials", ISSN 1313-0226, year IX, Issue $6 / 2015$, pp. 24-28.

[6] Minchev, C.N., Slavyanov, K.O., Neural network for font style letter symbol recognition in additive noise environment, "Machines, Technologies, Materials", ISSN 1313-0226. Issue 7/2013, pp. 24-28.

[7] Zadeh, L.A., The concept of a linguistic variable and its application to approximate reasoning-I, Information Sciences 8, 1975, pp. 199-249.

[8] Mamdani E.H., Assilian S., An experiment in linguistic synthesis with a fuzzy logic controller, International Journal of ManMachine Studies, Vol. 7, No. 1, 1975, pp. 1-13.

[9] Zadeh, L.A., Fuzzy logic, Computer ( Volume: 21, Issue: 4, April 1988 ), pp. $83-93$.

[10] Zadeh, L.A., Outline of a new approach to the analysis of complex systems and decision processes, IEEE Transactions on Systems, Man, and Cybernetics, Vol. 3, No. 1, Jan. 1973, pp. 28-44. 